\title{
A Greedy Algorithm for Constructing a Low-Width Generalized Hypertree Decomposition
}

\author{
Kaoru Katayama \\ Tokyo Metropolitan University \\ 6-6 Asahigaoka, Hino, Tokyo, \\ Japan 191-0065 \\ kaoru@tmu.ac.jp
}

\author{
Tatsuro Okawara \\ Tokyo Metropolitan University \\ 6-6 Asahigaoka, Hino, Tokyo, \\ Japan 191-0065 \\ okawara- \\ tatsuro@sd.tmu.ac.jp
}

\author{
Yuka Ito \\ Rakuten,Inc. \\ 4-12-3 Higashishinagawa, \\ Shinagawa, Tokyo, \\ Japan 140-0002 \\ yuka.a.ito@mail.rakuten.co.jp
}

\begin{abstract}
We propose a greedy algorithm which, given a hypergraph $H$ and a positive integer $k$, produces a hypertree decomposition of width less than or equal to $3 k-1$, or determines that $H$ does not have a generalized hypertree-width less than $k$. The running time of this algorithm is $O\left(m^{k+2} n\right)$, where $m$ is the number of hyperedges and $n$ is the number of vertices. If $k$ is a constant, it is polynomial. The concepts of (generalized) hypertree decomposition and (generalized) hypertree-width were introduced by Gottlob et al. Many important NP-complete problems in database theory or artificial intelligence are polynomially solvable for classes of instances associated with hypergraphs of bounded hypertree-width. Gottlob et al. also developed a polynomial time algorithm det-k-decomp which, given a hypergraph $H$ and a constant $k$, computes a hypertree decomposition of width less than or equal to $k$ if the hypertree-width of $H$ is less than or equal to $k$. The running time of det-k-decomp is $O\left(m^{2 k} n^{2}\right)$ in the worst case, where $m$ and $n$ are the number of hyperedges and the number of vertices, respectively. The proposed algorithm is faster than this. The key step of our algorithm is checking whether a set of hyperedges is an obstacle to a hypergraph having low generalized hypertree-width. We call such a local hypergraph structure a $k$-hyperconnected set. If a hypergraph contains a $k$-hyperconnected set with a size of at least $2 k$, it has hypertreewidth of at least $k$. Adler et al. propose another obstacle called a $k$-hyperlinked set. We discuss the difference between the two concepts with examples.
\end{abstract}

\section{INTRODUCTION}

The concepts of hypertree decomposition and hypertree-width were introduced by Gottlob et al. [5] Many important NP-complete problems in database theory and artificial intelligence such as the conjunctive query containment problem are polynomially solvable for classes of instances associated with hypergraphs of bounded hypertreewidth [5]. Gottlob et al. [7] also introduced the concept of generalized hypertree decomposition and generalized hypertree-width.

\footnotetext{
${ }^{*}$ A part of this work was completed while the author was a student at Tokyo Metropolitan University.
}

Permission to make digital or hard copies of all or part of this work for personal or classroom use is granted without fee provided that copies are not made or distributed for profit or commercial advantage and that copies bear this notice and the full citation on the first page. To copy otherwise, to republish, to post on servers or to redistribute to lists, requires prior specific permission and/or a fee.

ICDT 2010, March 22-25, 2010, Lausanne, Switzerland.

Copyright 2010 ACM 978-1-60558-947-3/10/0003 ...\$10.00
We propose a greedy algorithm which, given a hypergraph $H$ and a positive integer $k$, produces a hypertree decomposition of width less than or equal to $3 k-1$, or determines that $H$ does not have generalized hypertree-width less than $k$. Since a hypertree decomposition is also a generalized hypertree decomposition by definition, our algorithm produces a generalized hypertree decomposition. The running time of our algorithm is $O\left(m^{k+2} n\right)$, where $m$ is the number of hyperedges and $n$ is the number of vertices. If $k$ is a constant, the running time of our algorithm is polynomial. Gottlob et al. [9] also develped a polynomial time algorithm called det-k-decomp which, given a hypergraph $H$ and a positive integer $k$ as a constant, computes a hypertree decomposition of width less than or equal to $k$ if the hypertree-width of $H$ is less than or equal to $k$. If the hypertree-width of $H$ is more than $k, H$ is rejected. The running time of det-k-decomp is $O\left(m^{2 k} n^{2}\right)$ in the worst case and our algorithm is faster than det-k-decomp.

The key step of our algorithm is checking whether a set of hyperedges is an obstacle to a hypergraph with low generalized hypertreewidth. We call such a local hypergraph structure a $k$-hyperconnected set, where $k$ is a positive integer. We show that, if a hypergraph contains a $k$-hyperconnected set of size $2 k$, the generalized hypertreewidth of the hypergraph is at least $k$. If a given set of hyperedges is not a $k$-hyperconnected set, our algorithm finds a set of hyperedges called a separator, which separates two different subsets of the given set of hyperedges. This follows the approach used by Kleinberg and Tardos [12] for designing an algorithm for constructing a low-width tree decomposition of a graph. The tree decomposition algorithm runs in $O(f(k) m n)$ time, where $f(k)$ is a function that depends only on a positive integer $k$, and $m, n$ are the number of edges and vertices of a graph, respectively. In both algorithms, the running time is dominated by the time required to check whether a (hyper)graph contains an obstacle to a (hyper)graph having low (hyper)tree-width. In the tree decomposition algorithm, this can be done efficiently using an algorithm for network flow in $O(f(k) m)$ time. On the contrary, in our hypertree decomposition algorithm, it requires more time, $O\left(m^{k+1} n\right)$, because every possibility is checked.

Adler et al. [1] proposed another obstacle, a $k$-hyperlinked set, to a hypergraph with low generalized hypertree-width. A similar greedy algorithm to ours can be constructed with the concept of a $k$ hyperlinked set. We show the difference between a $k$-hyperconnected set and a $k$-hyperlinked set with examples. Although several algorithms for constructing a hypertree decomposition have already been proposed, as we mention in the next section, to our knowledge there is no other algorithm with the same approach to hypertree de- 
composition, which is trying to find an obstacle to a hypergraph having low generalized hypertree-width.

This paper is organized as follows: In Section 2, we discuss related work. In Section 3, we give definitions of hypergraphs and hypertree decompositions. In Section 4, we introduce the concept of a $k$-hyperconnected set as an obstacle to a low-width (generalized) hypertree decomposition and show the relation between the size of a $k$-hyperconnected set and the hypertree-width. We describe the algorithm check_k-hyperconnected which, given a hypergraph, a set of hyperedges and a positive integer $k$, checks whether the given set of hyperedges is a $k$-hyperconnected set. We also explain the difference between a $k$-hyperconnected set and a $k$-hyperlinked set with examples. Then, in Section 5, we introduce the algorithm low-width-ghd which, given a hypergraph and a positive integer $k$, constructs a (generalized) hypertree decomposition or reports that the hypergraph does not have the hypertree-width less than $k$. We also evaluate the running time of low-width-ghd. Finally, we conclude the paper in Section 6.

\section{RELATED WORK}

Gottlob et al. [5] proposed the alternating algorithm k-decomp, which, given a hypergraph $H$ a positive integer $k$, constructs a hypertree decomposition of minimal width less than or equal to $k$, if the hypertree-width of $H$ is less than or equal to $k$. If the hypertreewidth of $H$ is more than $k, \mathrm{k}$-decomp rejects $H$. They also presented the algorithm opt-k-decomp [6], which is another algorithm for computing a hypertree decomposition of minimal width less than or equal to $k$, given a hypergraph and a positive integer $k$. The running time of opt-k-decomp is $O\left(m^{2 k} n^{2}\right)$, where $m$ is the number of hyperedges and $n$ is the number of vertices. If $k$ is a constant, it is polynomial. Gottlob et al. [9] developed the algorithm det-k-decomp which, given a hypergraph $H$ and a positive integer $k$ as a constant, computes a hypertree decomposition of width less than or equal to $k$ if the hypertree-width of $H$ is less than or equal to $k$. If the hypertree-width of $H$ is more than $k, H$ is rejected. The running time of det-k-decomp is $O\left(m^{2 k} n^{2}\right)$ in the worst case, where $m$ and $n$ are the number of hyperedges and vertices in the hypergraph, respectively. Gottlob et al. [8] showed that deciding whether a hypergraph has generalized hypertree-width at most 3 is NP-complete.

Scarcello et al. [14] proposed modified versions of opt-k-decomp for computing a hypertree decomposition with cost functions. Dermaku et al. [2] used heuristics for generating tree decompositions and partitioning hypergraphs to produce hypertree decompositions. Harvey et al. [11] introduced the reduced normal form of a hypertree decomposition and improved opt-k-decomp.

Adler et al. [1] explored the relationship between hypertree width and various hypergraph invariants. Many structural decomposition methods of a hypergraph are proposed besides generalized hypertree decomposition. Grohe et al. [10] introduced the concept of fractional hypertree decomposition which is a generalization of generalized hypertree decomposition. Gottlob et al. [4] and Miklós [13] compared them.

\section{PRELIMINARIES}

We describe definitions of hypergraphs and (generalized) hypertree decompositions and introduce two properties of a (generalized) hypertree decomposition.

\subsection{Hypergraph}

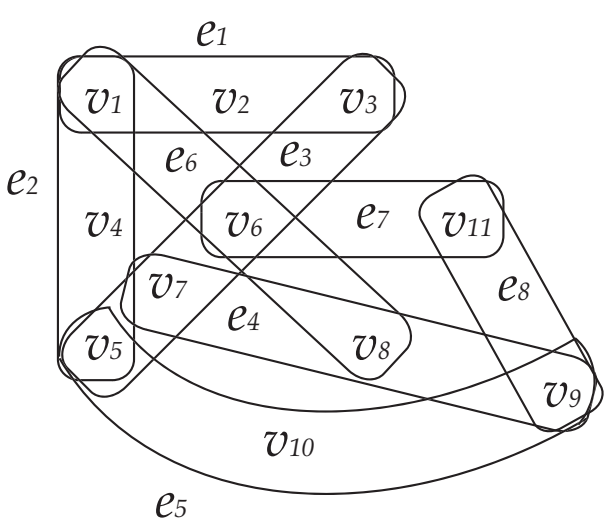

Figure 1: Connected hypergraph $H$

A hypergraph is a pair $H=(V(H), E(H))$, where $V(H)$ is a finite set of vertices and $E(H)$ is a set of hyperedges. A hyperedge is a subset of $V(H)$, which is not an empty set. We merely call a hyperedge an edge. For a set of edges $E \subseteq E(H)$, ver $(E)$ stands for $\bigcup_{e \in E} e$. We assume $\operatorname{ver}(E(H))=V(H)$.

Let $a$ and $b$ be two vertices in $V(H) . \quad a$ is adjacent to $b$ if an edge $e \in E(H)$ exists such that $\{a, b\} \subseteq e$. A path $(a, b)$ is a sequence $v_{0}(=a), v_{1}, v_{2}, \ldots, v_{h}(=b)$ of vertices such that $v_{i}$ is adjacent to $v_{i+1}(0 \leq i \leq h-1)$. A hypergraph $H$ is connected if, for any pair of two vertices $a, b \in V(H)$, a path $(a, b)$ exists. We deal with only connected hypergraphs in this paper. Let $W$ be a subset of $V(H) . a$ is [W]-adjacent to $b$ if an edge $e \in E(H)$ exists such that $\{a, b\} \subseteq e \backslash W$. A $[W]-p a t h(a, b)$ is a sequence $v_{0}(=$ $a), v_{1}, v_{2}, \ldots, v_{h}(=b)$ of vertices such that $v_{i}$ is $[W]$-adjacent to $v_{i+1}$ $(0 \leq i \leq h-1)$. A set of vertices $C \subseteq V(H)$ is [W]-connected if, for any pair of two vertices $a, b \in C$, there is a $[W]-\operatorname{path}(a, b)$. A [W]-component is a maximal $[W]$-connected non-empty set of vertices. Let $F$ be a subset of $E(H)$. A [F]-fragment is a maximal set of edges that share the vertices with a $[\operatorname{ver}(F)]$-component, that is, $\{e \in E(H) \mid e \cap[\operatorname{ver}(F)]$-component $\neq \emptyset\}$. For a set of vertices $C$, let a set of edges $\operatorname{cov}(C)$ be $\{e \in E(H) \mid e \cap C \neq \emptyset\}$, and a family of subsets of $\operatorname{cov}(C), \operatorname{cov}^{*}(C)$ be $\{F \subseteq \operatorname{cov}(C) \mid \forall e \in F: e \nsubseteq$ $\operatorname{ver}(\operatorname{cov}(C) \backslash e)\}$.

EXAMPLE 1. Consider connected hypergraph $H$ in Figure 1. The set of vertices $V(H)$ is $\left\{v_{1}, v_{2}, \ldots, v_{11}\right\}$ and the set of edges $E(H)$ is $\left\{e_{1}, e_{2}, \ldots, e_{8}\right\}$ where $e_{3}=\left\{v_{3}, v_{5}, v_{6}, v_{7}\right\}$ and $e_{6}=\left\{v_{1}, v_{6}, v_{8}\right\}$. For a set of vertices $W=\left\{v_{3}, v_{5}, v_{6}, v_{7}, v_{8}\right\}$, the [W]-components are $\left\{v_{1}, v_{2}, v_{4}\right\}$ and $\left\{v_{9}, v_{10}, v_{11}\right\}$. For a set of vertices $C=\left\{v_{9}, v_{10}, v_{11}\right\}$, a set of edges $\operatorname{cov}(C)$ is $\left\{e_{4}, e_{5}, e_{7}, e_{8}\right\}$ and a family of subsets of $\operatorname{cov}(C), \operatorname{cov}^{*}(C)$ is $\left\{\left\{e_{5}, e_{7}\right\},\left\{e_{5}, e_{8}\right\}\right\}$. For a set of edges $F=$ $\left\{e_{3}, e_{6}\right\}$, the $[F]$-fragments are $\left\{e_{1}\right\},\left\{e_{2}\right\}$ and $\left\{e_{4}, e_{5}, e_{7}, e_{8}\right\}$.

\subsection{Hypertree Decomposition}

A hypertree decomposition of a hypergraph $H$ is a triple $\langle T, \chi, \lambda\rangle$. $T=(V(T), E(T))$ is a rooted tree, where $V(T)$ is a finite set of nodes, and $E(T)$ is a set of edges of $T . \quad \chi: V(T) \rightarrow 2^{V(H)}$ and $\lambda: V(T) \rightarrow 2^{E(H)}$ are functions associating a set of vertices $\chi(t) \subseteq$ $V(H)$ and edges $\lambda(t) \subseteq E(H)$ to each node $t$ respectively. We call $v \in V(H)$ a vertex and $t \in V(T)$ a node. For any $t \in V(T), T^{t}$ denotes the maximal subtree of $T$ rooted at $t$. For a subtree $T^{\prime}$ of $T$, we use $\chi\left(T^{\prime}\right)$ and $\lambda\left(T^{\prime}\right)$ to denote $\bigcup_{n \in V\left(T^{\prime}\right)} \chi(n)$ and $\bigcup_{n \in V\left(T^{\prime}\right)} \lambda(n)$, 
respectively.

DEFINITION 1. (Hypertree Decomposition) [5] A hypertree decomposition of a hypergraph $H$ is a triple $\langle T, \chi, \lambda\rangle$, which satisfies all the following conditions:

1. for each edge e $\in E(H), t \in V(T)$ exists such that $e \subseteq \chi(t)$;

2. for each vertex $v \in V(H)$, the set $\{t \in V(T) \mid v \in \chi(t)\}$ induces a connected subtree of $T$;

3. for each $t \in V(T), \chi(t) \subseteq \operatorname{ver}(\lambda(t))$;

4. for each $t \in V(T)$, $\operatorname{ver}(\lambda(t)) \cap \chi\left(T^{t}\right) \subseteq \chi(t)$.

The width of a hypertree decomposition $\langle T, \chi, \lambda\rangle$ is the largest size of $\lambda(t)$ over every node $t$ of $T$. The hypertree-width of a hypergraph $H$ is the minimum width over all hypertree decompositions of $H$. The hypertree-width of an acyclic hypergraph is 1 .

A generalized hypertree decomposition of a hypergraph $H$ is a triple $\langle T, \chi, \lambda\rangle$, which satisfies conditions 1,2 , and 3 of Definition 1 . The width of a generalized hypertree decomposition $\langle T, \chi, \lambda\rangle$ is the largest size of $\lambda(t)$ over every node $t$ of $T$. The generalized hypertreewidth of a hypergraph $H$ is the minimum width over all generalized hypertree decompositions of $H$. The generalized hypertree-width of a hypergraph is less than or equal to the hypertree-width. [1].

DEFINITION 2. (Normal Form) [8] A generalized hypertree decomposition $\langle T, \chi, \lambda\rangle$ of a hypergraph $H$ is in normal form, if, for each vertex $t \in V(T)$ and each child s of $t$, all the following conditions hold:

1. there is exactly one $[\chi(t)]$-component $C_{t}$ such that $\chi\left(T^{s}\right)=$ $C_{t} \cup(\chi(s) \cap \chi(t))$;

2. $\chi(s) \cap C_{t} \neq \emptyset$, where $C_{t}$ is the $[\chi(t)]$-component satisfying condition 1;

3. $\operatorname{ver}(\lambda(s)) \cap \chi(t) \subseteq \chi(s)$.

The hypertree decomposition constructed with our algorithm is in normal form, as shown later in Proposition 7.

EXAMPLE 2. Figure 2 shows a normal form (generalized) hypertree decomposition of hypergraph $H$ in Figure 1. The width of this (generalized) hypertree decomposition is 2.

A hypergraph is separated by deleting vertices assigned to a node or common vertices assigned to two connected nodes in the (generalized) hypertree decomposition.

Proposition 1. Suppose that there are subtrees $T_{1}, T_{2}, \ldots, T_{d}$ when a node $p$ is deleted from tree $T$ of a (generalized) hypertree decomposition $\langle T, \chi, \lambda\rangle$ of a hypergraph $H$. Then for any pair $i, j \in$ $\{1,2, \ldots, d\}(i \neq j),\left(\chi\left(T_{i}\right) \backslash \chi(p)\right) \cap\left(\chi\left(T_{j}\right) \backslash \chi(p)\right)=\emptyset$ and $\{e \in$ $\left.E(H) \mid\{u, v\} \subseteq e, u \in \chi\left(T_{i}\right) \backslash \chi(p), v \in \chi\left(T_{j}\right) \backslash \chi(p)\right\}=\emptyset$ (Figure 3).

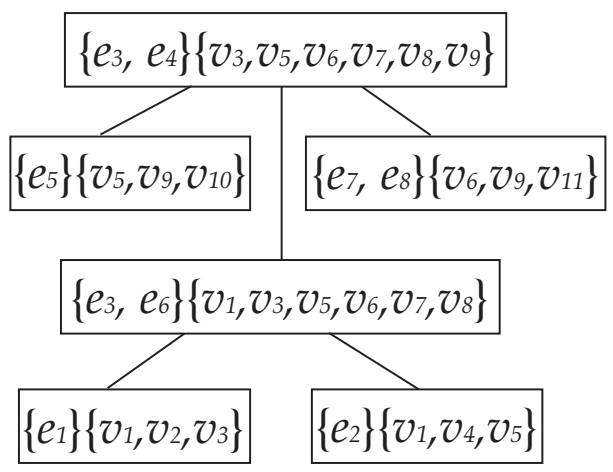

Figure 2: Normal form hypertree decomposition of $H$

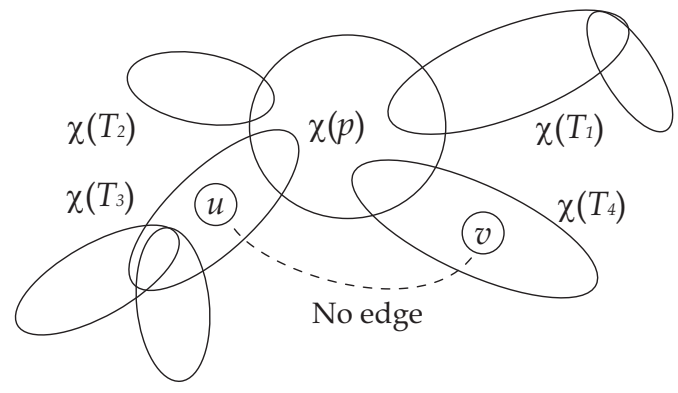

Figure 3: Subtrees $T_{1}, T_{2}, \ldots, T_{d}$ by deleting node $p$ from a (generalized) hypertree decomposition. There is no edge which contains vertices $u$ and $v$ when $\chi(p)$ is deleted from hypergraph.

Proof. Omitted.

Proposition 2. Suppose that there are subtrees $T_{p}$ and $T_{t}$ when an edge $(p, t) \in E(T)(p, t \in V(T))$ is deleted from tree $T$ of a (generalized) hypertree decomposition $\langle T, \chi, \lambda\rangle$ of a hypergraph $H$. Then by deleting $\chi(p) \cap \chi(t)$ from $H, H$ is disconnected into two components, $\chi\left(T_{p}\right) \backslash(\chi(p) \cap \chi(t))$ and $\chi\left(T_{t}\right) \backslash(\chi(p) \cap \chi(t))$. That is, $\left(\chi\left(T_{p}\right) \backslash(\chi(p) \cap \chi(t))\right) \cap\left(\chi\left(T_{t}\right) \backslash(\chi(p) \cap \chi(t))\right)=\emptyset$ and $\left\{e \in E(H) \mid\{u, v\} \subseteq e, u \in \chi\left(T_{p}\right) \backslash(\chi(p) \cap \chi(t)), v \in \chi\left(T_{t}\right) \backslash(\chi(p) \cap\right.$ $\chi(t))\}=\emptyset$ (Figure 4).

ProOF. Omitted.

\section{OBSTACLES TO LOW GENERALIZED HYPERTREE-WIDTH}

The key step in designing our algorithm is trying to find an obstacle to a hypergraph having low generalized hypertree-width. We call such an obstacle a k-hyperconnected set, which is a set of edges of the hypergraph. The notion of a k-hyperconnected set is an adaptation of $k$-connectedness for a graph to our setting [3]. We show the relation between the size of a $k$-hyperconnected set in a hypergraph and the hypertree-width of the hypergraph. We propose the algorithm check_k-hyperconnected to decide whether a subset of edges of a hypergraph is a $k$-hyperconnected set, given a hypergraph and a positive integer $k$. The running time of $k$ hyperconnected set is $O\left(m^{k+1} n\right)$. If $k$ is a constant, it is polynomial. 


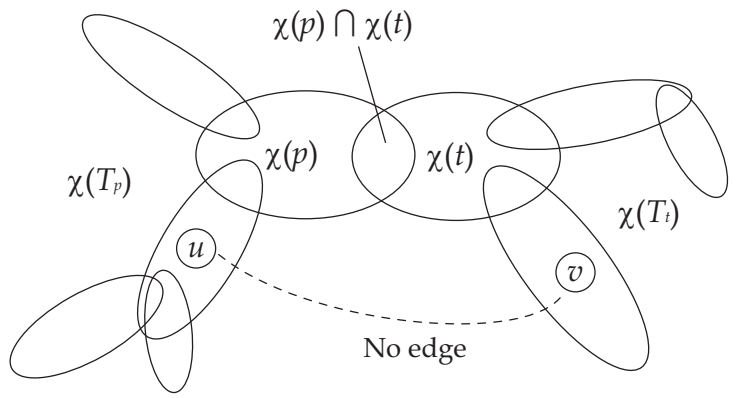

Figure 4: Subtrees $T_{p}$ and $T_{t}$ by deleting the edge between node $p$ and node $t$ from a (generalized) hypertree decomposition. There is no edge which contains vertices $u$ and $v$ when $\chi(p) \cap \chi(t)$ is deleted from hypergraph.

\section{1 $k$-hyperconnected set}

We give the definition of a $k$-hyperconnected set and prove a proposition for its algorithmic use.

DEFINITION 3. (separator) Let $Y$ and $Z$ be a pair of subsets of $E(H)$ of a hypergraph $H$ such that $|Y|=|Z|$ and $Y \neq Z$. A subset of $E(H), S$ is a separator for a pair of $Y$ and $Z$ if it satisfies all the following conditions:

1. $|S|<|Y|=|Z|$;

2. there is no [ver $(S)]$-path from ver $(Y)$ to ver $(Z)$.

We say that $S$ separates $Y$ and $Z$, or that $Y$ and $Z$ are separable with $S$.

DEFINITION 4. (k-hyperconnected set) Let $X$ be a subset of $E(H)$ of a hypergraph $H$ and $k$ be a positive integer. Let $Y$ and $Z$ be an arbitrary pair of two subsets of $X$ such that $|Y|=|Z| . X$ is a $k$-hyperconnected set, if it satisfies all the following conditions:

1. $|X| \geq k$;

2. $X$ does not contain separable subsets $Y$ and $Z$, where $|Y|=$ $|Z| \leq k$. In other words, there is no separator $S \subseteq E(H)$, which separates $Y$ and $Z$ such that $|S|<|Y|=|Z| \leq k$.

We call an edge, which is included in X, an X-edge.

Intuitively, a $k$-hyperconnected set is highly self-entwined. It does not have any small parts that can easily split off from each other. A $k$-hyperconnected set cannot be separated by deleting less than $k$ edges.

PROPOSITION 3. If a hypergraph $H$ contains a k-hyperconnected set with a size of at least $2 k, H$ has the generalized hypertree width of at least $k$.

Proof. Suppose that a hypergraph $H$ contains a $k$-hyperconnected set $X$ with a size of at least $2 k$, and it has a generalized hypertree decomposition $\langle T, \chi, \lambda\rangle$ of a width less than $k$. There is a node $t$ of $T$ that satisfies the following conditions:
1. Let $X^{t}$ be a subset of $X$-edges $\left\{x \in X \mid x \subseteq \chi\left(T^{t}\right)\right\}$. $\left|X^{t}\right|$ is more than or equal to $\left\lceil\frac{|X|}{2}\right\rceil$;

2. $t$ is as far from the root of $T$ as possible.

Clearly, $\chi(t)$ contains all vertices of at least one $X$-edge, and node $t$ is not a leaf of $T$ because the set of edges $X$ with a size of at least $2 k$ cannot be contained in a node of the generalized hypertree decomposition of a width less than $k$. Now we divide $X$ into three distinct subsets, $X_{p}=X \backslash X^{t}, X_{t}=\{x \in X \mid x \subseteq \chi(t)\}$, and $X_{c}=X^{t} \backslash$ $X_{t}$. There is no $[\chi(t)]$-path between any pair of vertices in $X_{p}$ and $X_{c}$ from Proposition 1. The size of $X_{p}$ and $X_{c}$ is less than or equal to $k$. Two subsets, $Y$ and $Z$, of $E(H)$, where $\left|X_{t}\right|<|Y|=|Z| \leq k$, can be made from $X_{p}$ and $X_{c}$ by adding edges in $X_{t}$. Then $X_{t}$ separates $Y$ and $Z$. This means that $X$ is not a $k$-hyperconnected set and contradicts the assumption.

\subsection{Comparing with $k$-hyperlinked set}

Adler et al. [1] define the concept of a k-hyperlinked set for a set of edges of a hypergraph. Hyperlinkedness of a hypergraph is the largest integer $k$ for which the hypergraph contains a $k$-hyperlinked set. It is an adaptation of the linkedness of a graph. A $k$-hyperlinked set also an obstacle to a hypergraph having low generalized hypertreewidth. We show that the size of a $k$-hyperlinked set is also associated with the generalized hypertree-width of the hypergraph, and compare the two notions using examples. Adler et al. [1] prove that the hyperlinkedness of a hypergraph is less than or equal to the generalized hypertree-width of the hypergraph.

DEFINITION 5. (X-big) [1] Let $H$ be a hypergraph and $X$ be a subset of $E(H)$. A subset of vertices $V(H), C$ is $X$-big, if it satisfies the following condition:

$$
|\{e \in X \mid e \cap C \neq \emptyset\}|>\frac{|X|}{2} .
$$

An $X$-big component is a maximal set of $X$-big vertices in which each vertex is adjacent to another one.

DEFINITION 6. (k-hyperlinked set) [1] Let $H$ be a hypergraph and $k$ be a positive integer. A subset of $E(H), X$ is a $k$-hyperlinked set, if the hypergraph $(V(H) \backslash \operatorname{ver}(S),\{e \cap(V(H) \backslash \operatorname{ver}(S)) \mid e \in E(H)\})$ has an $X$-big component for any set $S \subseteq E(H)$ where $|S|<k$. We call an edge, which is included in $X$, an $X$-edge as in Definition 4.

PROPOSITION 4. If a hypergraph $H$ contains a k-hyperlinked set with a size of at least $2 k, H$ has a generalized hypertree width of at least $k$.

Proof. This proposition can be proven by the same idea of Proposition 3.

We show the difference between a $k$-hyperlinked set and a $k$-hyperconnected set with the following examples.

EXAMPLE 3. A hypergraph $H$ and a subset $X=X_{1} \cup X_{2}$ of $E(H)$ are defined as in Figure 5. In this case $X$ is a 1-hyperconnected 


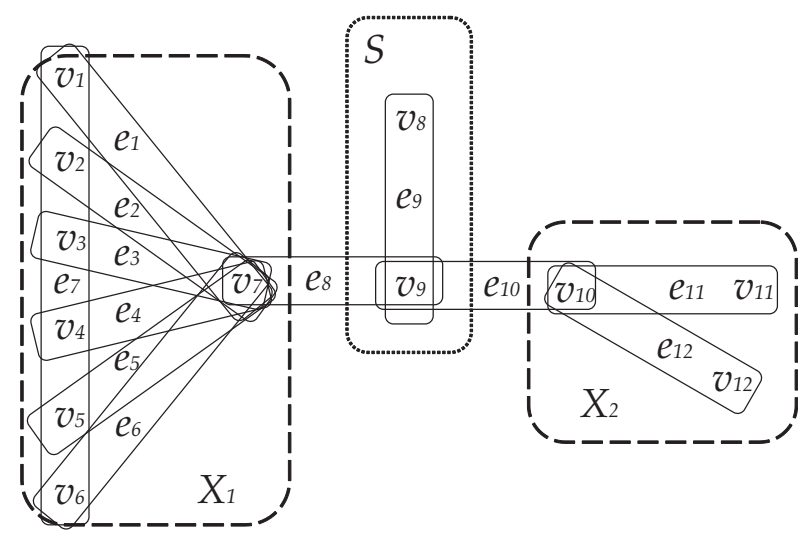

Figure 5: Hypergraph for example 3. $X=X_{1} \cup X_{2}$ is a 1hyperconnected set and a 2-hyperlinked set.

set and a 2-hyperlinked set. Let two sets of edges, $Y$ and $Z$, such that $|Y|=|Z|=2$ be subsets of $X_{1}$ and $X_{2}$, respectively. Since there is no $[\operatorname{ver}(S)]$-paths $(y, z)$ for any pair of vertices, $y \in Y$ and $z \in Z, X$ is a 1-hyperconnected set. On the other hand, in a hyper$\operatorname{graph}(V(H) \backslash e,\{e \cap(V(H) \backslash e) \mid e \in E(H)\})$ constructed by deleting any edge $e \in E(H)$ from $H$, the number of remaining edges in $X$ is larger than $|X| / 2=9 / 2$. But, in a hypergraph $\left(V(H) \backslash\left(e_{1} \cup\right.\right.$ $\left.\left.e_{7}\right),\left\{e \cap\left(V(H) \backslash\left(e_{1} \cup e_{7}\right)\right) \mid e \in E(H)\right\}\right)$ constructed by deleting two edges, $e_{1}$ and $e_{7}$, from $H$, the number of remaining edges in $X$ is 2 and less than $|X| / 2=9 / 2$. This means that $X$ is a 2-hyperlinked set.

EXAMPLE 4. A hypergraph $H$ and a subset $X$ of $E(H)$ are defined as in Figure 6. In this case $X$ is a 3-hyperconnected set and a 2-hyperlinked set. Any two subsets $Y$ and $Z$ each of size 3 of $X$ cannot be separated by deleting any set of edges of size 2. This means that $X$ is a 3-hyperconnected set at least. Since there are no two different subsets each of size 4 of $X$, we cannot choose separable subsets for a separator of size 3. Therefore $X$ is not a 4-hyperconnected set. On the other hand, in a hypergraph $(V(H) \backslash e,\{e \cap(V(H) \backslash e) \mid e \in E(H)\})$ constructed by deleting any edge $e \in E(H)$ from $H$, the number of remaining edges in $X$ is larger than $|X| / 2=2$. But, in a hypergraph $\left(V(H) \backslash\left(e_{2} \cup e_{3}\right),\{e \cap\right.$ $\left.\left.\left(V(H) \backslash\left(e_{2} \cup e_{3}\right)\right) \mid e \in E(H)\right\}\right)$ constructed by deleting two edges, $e_{2}$ and $e_{3}$ from $H$, the number of remaining edges in $X$ is $|X| / 2=2$. This means that $X$ is a 2-hyperlinked set.

\subsection{Finding Separator}

We describe the algorithm check_k-hyperconnected which, given a hypergraph $H$, a subset $X$ of edges of $H$ and a positive integer $k$, determines whether $X$ is a $k$-hyperconnected set. If $X$ is not a $k$ hyperconnected set, check_k-hyperconnected returns a set of edges as a separator for a pair of two separable subsets in $X$. We can develop a similar algorithm using the notion of a $k$-hyperlinked set.

A simple way to do this is to check whether there is a separator for every pair of subsets of each size less than or equal to $k$ of $X$. However, it is not easy to find such a separator. Therefore, we check whether a pair of separable subsets, $Y$ and $Z$, of $X$ exists for every subset with a size of less than $k$ of $E(H)$ conversely. If the size of $X$ is more than or equal to $2 k-1$, it is necessary to check it

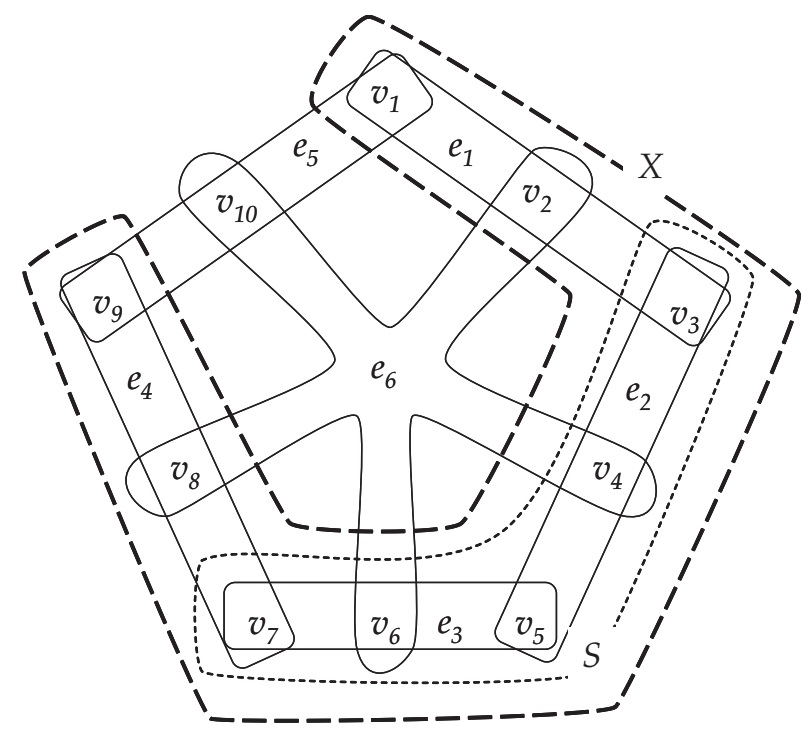

Figure 6: Hypergraph for example 4. $X$ is a 3-hyperconnected set and a 2-hyperlinked set.

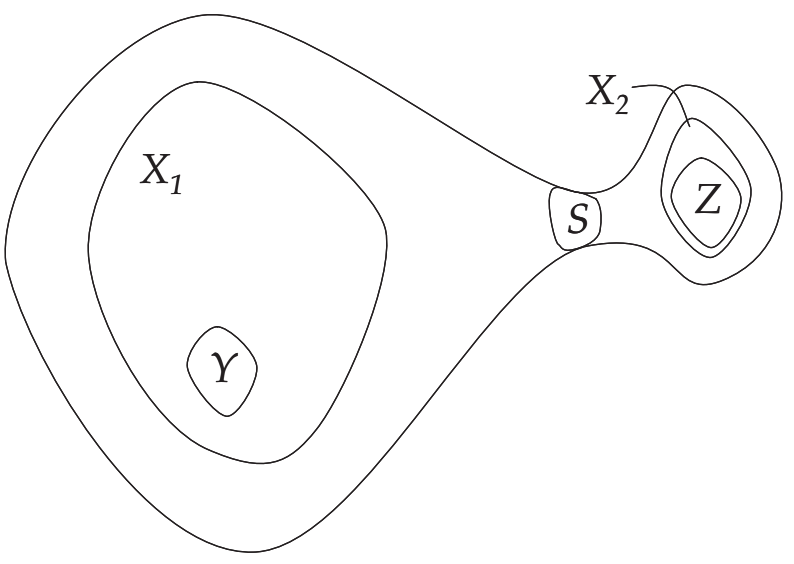

Figure 7: $X$-edges in $X \backslash(Y \cup Z \cup S)$ are added to $S$ to make the size of $S$ be $k-1$. Edges in $S \cap X$ are added to $Y$ and $Z$ to make their size be $k$.

only for every subset of size $k-1$ of $E(H)$ because if a separator with a size of less than $k-1$ is found, we can make it be $k-1$ by adding edges in $X \backslash(Y \cup Z \cup S)$ (Figure 7). That is, every separator is contained in subsets of size $k-1$ of $E(H)$. In the case where the size of a separator $S$ is $k-1$, each size of separable subsets $Y$ and $Z$ must be more than $k-1$ to satisfy the first condition in Definition 3. When candidate sets $Y$ and $Z$ for separable subsets are found for a subset $S$ of size $k-1$ of $E(H)$, but each size of $Y$ and $Z$ is less than or equal to $k-1$, we may increase the size by adding the same edges in $S \cap X$ to $Y$ and $Z$ (Figure 7). If we can make the size be $k$, the set of edges, $Y, Z$, and $S$ become separable subsets and the separator.

check_k-hyperconnected repeats the following steps for every subset $S$ of size $k-1$ of $E(H)$, as shown in Algorithm 1, unless it finds a separator of size $k-1$ or that a given $X$ is a $k$ hyperconnected set, that is, $X$ does not contain separable subsets $Y$ and $Z$ of each size less than or equal to $k$. We do not check whether the size of $X$ is more than or equal to $2 k-1$ since the size of $X$ is al- 
ways more than $2 k$ in the algorithm using check_k-hyperconnected, which constructs a low-width (generalized) hypertree decomposition. In Algorithm 1 , we use variables $Y, Z$, and $S$ to denote candidates of two separable subsets of $X$ and a separator for the subsets, respectively.

1. Choose a subset $S$ of size $k-1$ from $E(H)$.

2. Let $L$ be $\{e \in X \mid e \subseteq \operatorname{ver}(S)\}$. If $|L| \geq k$, return the subset $S$ as a separator and stop.

Any edge $e$ in $L$ can belong to both $Y$ and $Z$ because $e \backslash \operatorname{ver}(S)$ is an empty set, and there is no $[\operatorname{ver}(S)]$-path from $e$ to other vertices. Therefore, if $|L| \geq k$, we can construct $Y, Z$, and $S$, which satisfy the conditions of Definition 3, by choosing $k$ edges from $L$ as $Y, k$ edges from $X$ as $Z$, and the subset $S$ as a separator.

3. Divide the set of the $[S]$-fragments into two subsets, $Y$ and $Z$.

4. If there are more than or equal to $k X$-edges in each of $Y \cup L$ and $Z \cup L$, return the subset $S$ as a separator and stop.

If each $Y \cup L$ and $Z \cup L$ includes more than or equal to $k X$ edges, we can make separable subsets $Y^{\prime}$ and $Z^{\prime}$, which satisfy the conditions of Definition 3, by choosing $k$ edges from $L$ as $Y^{\prime}$, and $k$ edges from $X$ as $Z^{\prime}$. In this case, the subset $S$ separates $Y^{\prime}$ and $Z^{\prime}$.

PROPOSITION 5. The running time of check_k-hyperconnected is $O\left(\left(\begin{array}{c}m \\ k-1\end{array}\right) m^{2} n\right)$.

PROOF. Let $k$ be a positive integer as a constant, and $m, n$ be the number of edges $|E(H)|$ of a hypergraph $H$ and the number of vertices $|V(H)|$, respectively. The number of subsets of $E(H)$, where each of their sizes is $k-1$, is $\left(\begin{array}{c}m \\ k-1\end{array}\right)$. For each subset $S$ of size $k-1$ of $E(H)$, we enumerate the number of edges $\{e \in X \mid e \subseteq \operatorname{ver}(S)\}$. This takes $O(m n)$ time. Finding the set of $[S]$-fragments and dividing it into two subsets take $O\left(m^{2} n\right)$ time. Thus, the whole running time of check_k-hyperconnected is $O\left(\left(\begin{array}{c}m \\ k-1\end{array}\right) m^{2} n\right)$. Since $O\left(\left(\begin{array}{c}m \\ k-1\end{array}\right)\right)$ is $O\left(m^{k-1}\right)$, a less accurate but more readable upper bound of the running time is $O\left(m^{k+1} n\right)$.

\section{CONSTRUCTING A LOW-WIDTH HYPER- TREE DECOMPOSITION}

We propose an algorithm for constructing a (generalized) hypertree decomposition of $H$ of width less than or equal to $3 k-1$ or detemines that $H$ does not have a generalized hypertree-width less than $k$, where $k$ is a positive integer as a constant. The following procedure repeatedly decomposes a hypergraph by deleting a set of edges and constructs a (generalized) hypertree decomposition $\langle T, \chi, \lambda\rangle$. The proposed algorithm is described formally in Algorithm 2 and 3 . Figure 8 and 9 show decomposed components of a hypergraph, and Figure 10 shows the constructed hypertree decomposition corresponding to Figure 8 and 9.

1. Arbitrarily select a set of edges less than or equal to $2 k-1$ from $E(H)$ and make the root $r$ of $T$.

For the root $r$ of $T$, the selected set of edges is assigned to $\lambda(r)$, and all vertices included in the edges are assigned to $\chi(r)$. In Figure 10, a set of edges $E$ and a set of vertices $\chi(E)$ are assigned to $\lambda(r)$ and $\chi(r)$, respectively.

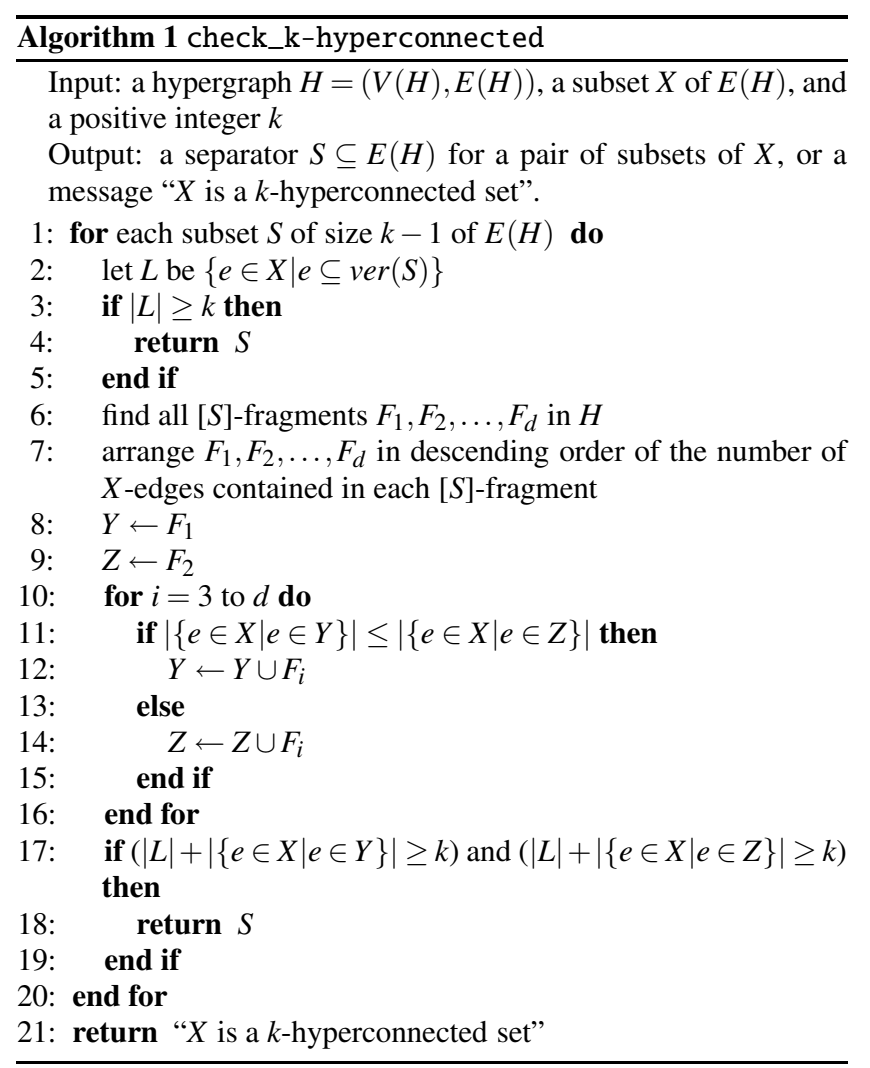

2. For each $[\chi(r)]$-component $C_{r}$, make a child node $t$ of the root $r$.

By Proposition 1 and 2, we can deal with each $[\chi(r)]$-component $C_{r}$ independently. Figure 8 shows that there are two $[\chi(r)]$ components $C_{r_{1}}$ and $C_{r_{2}}$. To decompose a $[\chi(r)]$-component $C_{r}$ further, we choose an arbitrary edge $e_{t}$ from $\operatorname{cov}\left(C_{r}\right)$. For child node $t$ corresponding to $C_{r}$, we add the edge $e_{t}$ and $e_{t}$ to $\lambda(t)$ and $\chi(t)$ respectively. To ensure that condition 2 of Definition 1 is satisfied when some vertices in $B_{r}=\operatorname{ver}\left(\operatorname{cov}\left(C_{r}\right)\right) \cap \chi(r)$ are included in a child node of $t$ in the later process, we add vertices $B_{r}$ to $\chi(t)$. Figure 8 shows that there are four vertices in $B_{r_{1}}$. We also add a set of edges $E_{A_{r}} \in \operatorname{cov}^{*}\left(A_{r}\right)$ where $A_{r}=B_{r} \backslash e_{t}$, and a set of vertices $\operatorname{ver}\left(E_{A_{r}}\right)$ to $\lambda(t)$ and $\chi(t)$, respectively, to satisfy condition 3 of Definition 1. Figure 8 shows that there are three vertices in $A_{r_{1}}$ and two edges in $E_{A_{r_{1}}}$. In Figure 10, $B_{r_{1}}$ is not contained in $\chi(t)$ since it is included in $e_{t} \cup \operatorname{ver}\left(E_{A_{r_{1}}}\right)$. Since $\operatorname{ver}(\lambda(t))$ is equal to $\chi(t)$, condition 4 of Definition 1 is also satisfied.

3. For each $[\chi(r) \cup \chi(t)]$-component $C_{t}$ formed from a $[\chi(r)]$ component $C_{r}$, make a child node $s$ of $t$ in the same way to step 2 above.

Figure 9 shows that there are three $[\chi(r) \cup \chi(t)]$-component $C_{t_{1}}, C_{t_{2}}, C_{t_{3}}$ formed from an $[\chi(r)]$-component $C_{r_{1}}$.

The tree $\langle T, \chi, \lambda\rangle$ constructed from the above procedure satisfies all the conditions of Definition 1 and is a (generalized) hypertree decomposition.

To determine whether the hypertree decomposition of the required size can be constructed, for each child node of $r$, we check the size 


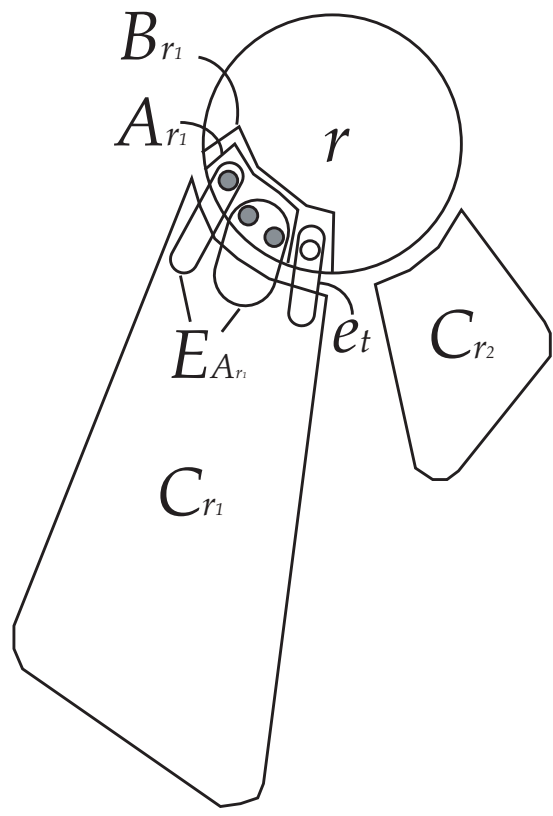

Figure 8: $[\chi(r)]$-components $C_{r 1}$ and $C_{r 2} . B_{r_{1}}$ is vertices in $\chi(r)$ that are also contained in $\operatorname{ver}\left(C_{r_{1}}\right) . A_{r_{1}}$ is vertices in $B_{r_{1}}$ that is not included in $e_{t}$. $E_{A_{r_{1}}}$ is element of $\operatorname{cov}^{*}\left(A_{r_{1}}\right)$.

of $\lambda(t)=\left\{e_{t}\right\} \cup E_{A_{r}}$ after the above step 2 . Here, there is clearly a set of edges $E_{A_{r}} \in \operatorname{cov}^{*}\left(A_{r}\right)$ less than or equal to $2 k-1$ because $A_{r} \subseteq \operatorname{ver}(\lambda(r))$ and $|\lambda(r)| \leq 2 k-1$. If the size of $\lambda(t)$ is less than or equal to $2 k-1$, node $t$ can be treated the same as root $r$, and we go through the procedure. If the size of $\lambda(t)$ is $2 k$, we check whether $\lambda(t)$ is a $k$-hyperconnected set with check-k_hyperconnected described in Section 4. There are the following two cases.

- $\lambda(t)=\left\{e_{t}\right\} \cup E_{A_{r}}$ is a $k$-hyperconnected set

The hypergraph does not have a generalized hypertree-width less than $k$ by Proposition 3. hd-decomp returns the message and halts.

- $\lambda(t)=\left\{e_{t}\right\} \cup E_{A_{r}}$ is not a $k$-hyperconnected set

There is a separator $S \subseteq E(H)$ of size $k-1$ and two separable sets of edges $Y, Z \subseteq \lambda(t)$ of size $k$ each. Figure 11 shows this situation in a $[\chi(r)]$-component. To decompose the $[\chi(r)]-$ component, we add $S \cap \operatorname{cov}\left(C_{r}\right)$ to $\lambda(t)$ and $\operatorname{ver}\left(S \cap \operatorname{cov}\left(C_{r}\right)\right)$ to $\chi(t)$. Since the size of $S \cap \operatorname{cov}\left(C_{r}\right)$ is less than the size of $S$, the size of $\lambda(t)=E_{A_{r}} \cup\left\{e_{t}\right\} \cup\left(S \cap \operatorname{cov}\left(C_{r}\right)\right)$ is less than or equal to $3 k-1$, which is the width we want.

To continue to the same process further for each $[\chi(r) \cup$ $\chi(t)]$-component $C_{t}$, the size of $E_{A_{t}} \in \operatorname{cov}^{*}\left(A_{t}\right)$ needs to be less than or equal to $2 k-1$ as the size of $E_{A_{r}}$. Since there is no $[\chi(S)]$-path between $[\chi(r) \cup \chi(t)]$-components, a set of vertices ver $\left(\operatorname{cov}\left(C_{t}\right)\right)$ has common vertices with either $\operatorname{ver}(Y \cup$ $S)$ or $\operatorname{ver}(Z \cup S)$ (Figure 11). $A_{t}$ is a subset of $\operatorname{ver}\left(\operatorname{cov}\left(C_{t}\right)\right)$. Therefore $\operatorname{cov}^{*}\left(A_{t}\right)$ is included in a subset of either $Y \cup S$ or $Z \cup S$. Since both size of $Y \cup S$ and $Z \cup S$ is less than or equal to $2 k-1$, the size of $E_{A_{t}} \in \operatorname{cov}^{*}\left(A_{t}\right)$ is also less than or equal to $2 k-1$.
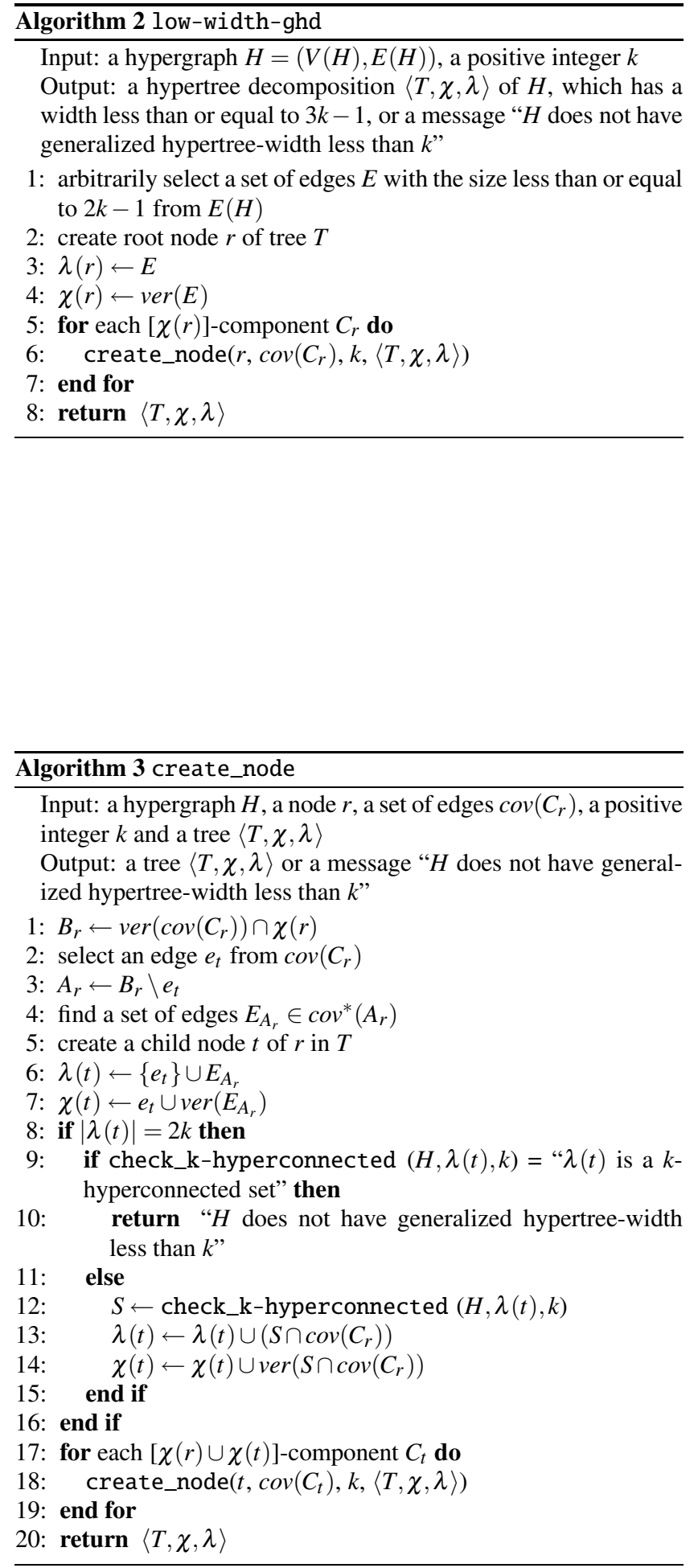


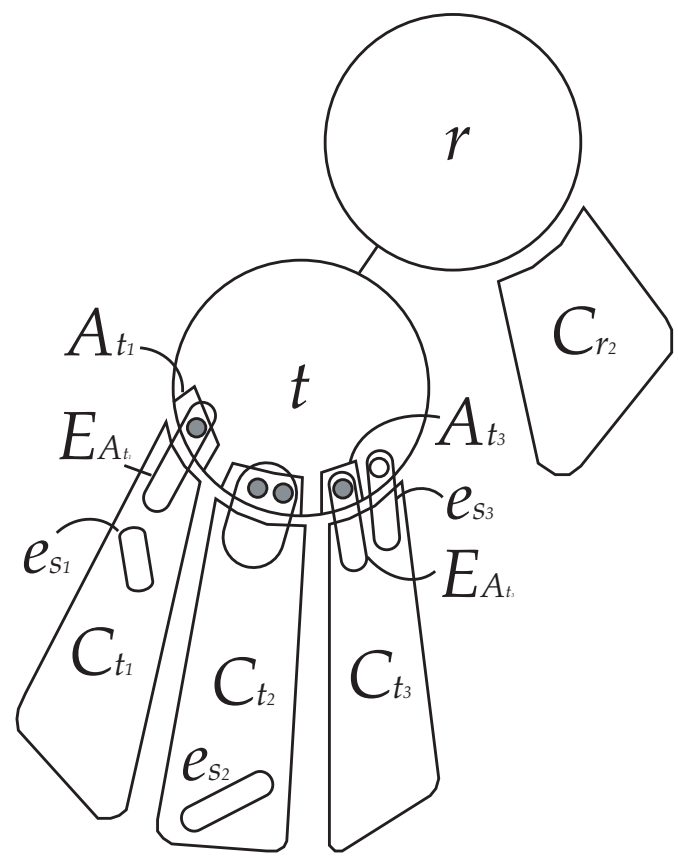

Figure 9: $C_{t_{1}}, C_{t_{2}}$, and $C_{t_{3}}$ are $[\chi(r) \cup \chi(t)]$-components formed from $C_{r_{1}}$.

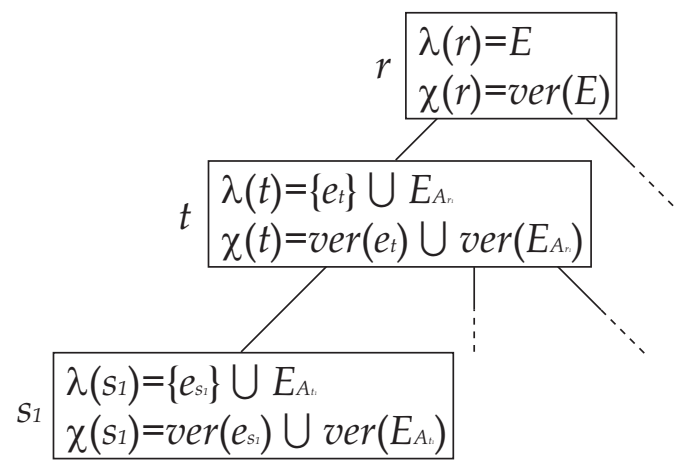

Figure 10: Hypertree decomposition for Figure 8 and 9.

PROPOSITION 6. The running time of low-width-ghd is $O\left(m^{k+2} n\right)$.

ProOF. Let $k$ be a positive integer, $m$ be the number of edges in a hypergraph $H$, and $n$ be the number of the vertices. The most costly operation in low-width-ghd is check_k-hyperconnected in create_node. Since one edge $e \in E(H)$ is selected at most once in create_node, create_node is called at most $m$ times. From Proposition 6, check_k-hyperconnected takes $O\left(m^{k+1} n\right)$. Thus, the entire running time of low-width-ghd is $O\left(m^{k+2} n\right)$.

PROPOSITION 7. A hypertree decomposition constructed by low-width-ghd is in normal form.

PROOF. low-width-ghd creates a child node $s$ of $t \in V(T)$ for each $[\chi(t)]$-component and assigns $e_{s} \cup \operatorname{ver}\left(E_{A_{t}}\right)$ to $\chi(s)$ in create_node, where $e_{s}$ is selected arbitrary from $\operatorname{cov}\left(C_{t}\right)$ and $A_{t}$ is $\left(\operatorname{ver}\left(\operatorname{cov}\left(C_{t}\right)\right) \cap \chi(t)\right) \backslash e_{s}$. Thus, conditions 1 and 2 of Definition 2 are clearly satisfied. Since $\chi(s)$ contains all vertices of

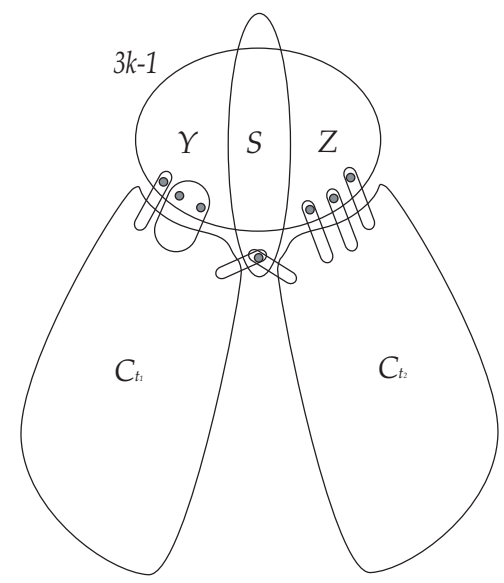

Figure 11: $[\chi(r) \cup \chi(t)]$-component $C_{t_{1}}$ and $C_{t_{2}}$ have common vertices with $\operatorname{ver}(Y \cup S)$ and $\operatorname{ver}(Z \cup S)$, respectively, where $S$ separates $Y$ and $Z$.

$\lambda(s)$ which consists of $\left\{e_{s}\right\}, E_{A_{t}}$ and, if $\lambda(t)$ of size $2 k$ is not a $k$-hyperconnected set, $S \cap \operatorname{cov}\left(C_{t}\right)$ where $S$ is a separator of $\lambda(t)$, condition 3 of Definition 2 is also satisfied.

\section{CONCLUSIONS}

We have presented a greedy algorithm which, given a hypergraph $H$ and a positive integer $k$ as a constant, produces a hypertree decomposition of a width less than or equal to $3 k-1$, or reports that $H$ does not have a generalized hypertree-width of less than $k$. The key step of this algorithm is trying to find a $k$-hyperconnected set, which is an obstacle to a hypergraph having a low generalized hypertree-width. The entire running time is $O\left(m^{k+2} n\right)$ where $m$ is the number of edges and $n$ is the number of vertices in a hypergraph. If $k$ is a constant, it is polynomial. This algorithm is faster than det-k-decomp developed by Gottlob et al. in the worst case.

\section{ACKNOWLEDGEMENTS}

The authors thank the anonymous reviewers for their helpful feedback. This work was supported by Grant-in-Aid for Scientific Re$\operatorname{search}(\mathrm{C})(21500104)$.

\section{REFERENCES}

[1] I. Adler, G. Gottlob, and M. Grohe. Hypertree width and related hypergraph invariants. Eur. J. Comb., 28(8):2167-2181, 2007.

[2] A. Dermaku, T. Ganzow, G. Gottlob, B. J. McMahan, N. Musliu, and M. Samer. Heuristic methods for hypertree decomposition. In 7th Mexican International Conference on Artificial Intelligence(MICAI), pages 1-11, 2008.

[3] R. Diestel. Graph Theory Second Edition. Springer, 2000.

[4] G. Gottlob, N. Leone, and F. Scarcello. A comparison of structural csp decomposition methods. In IJCAI' '99: Proceedings of the Sixteenth International Joint Conference on Artificial Intelligence, pages 394-399, San Francisco, CA, USA, 1999. Morgan Kaufmann Publishers Inc.

[5] G. Gottlob, N. Leone, and F. Scarcello. Hypertree decompositions and tractable queries. In PODS '99: Proceedings of the eighteenth ACM SIGMOD-SIGACT-SIGART symposium on Principles of database systems, pages 21-32, New York, NY, USA, 1999. ACM. 
[6] G. Gottlob, N. Leone, and F. Scarcello. On tractable queries and constraints. In 10 th International Conference and Workshop on Database and Expert Systems Applications(DEXA), pages 1-15, 1999.

[7] G. Gottlob, N. Leone, and F. Scarcello. Robbers, marshals, and guards: game theoretic and logical characterizations of hypertree width. In PODS '01: Proceedings of the twentieth ACM SIGMOD-SIGACT-SIGART symposium on Principles of database systems, pages 195-206, New York, NY, USA, 2001. ACM.

[8] G. Gottlob, Z. Miklós, and T. Schwentick. Generalized hypertree decompositions: Np-hardness and tractable variants. J. ACM, 56(6):1-32, 2009.

[9] G. Gottlob and M. Samer. A backtracking-based algorithm for hypertree decomposition. J. Exp. Algorithmics, 13:1.1-1.19, 2009.

[10] M. Grohe and D. Marx. Constraint solving via fractional edge covers. In SODA '06: Proceedings of the seventeenth annual ACM-SIAM symposium on Discrete algorithm, pages 289-298, New York, NY, USA, 2006. ACM.

[11] P. Harvey and A. Ghose. Reducing redundancy in the hypertree decomposition scheme. In IEEE International Conference on Tools with Artificial Intelligence (ICTAI), pages 474-481. IEEE Computer Society, 2003.

[12] J. Kleinberg and E. Tardos. Algorithm Design. Addison Wesley, 2006.

[13] Z. Miklós. On the parallel complexity of structural CSP decomposition methods. In H. Broersma, S. Dantchev, M. Johnson, and S. Szeider, editors, Algorithms and Complexity in Durham 2007, Proceedings of the third ACiD Workshop, volume 9 of Texts in Algorithmics, pages 107-118. College Publications London, 2007.

[14] F. Scarcello, G. Greco, and N. Leone. Weighted hypertree decompositions and optimal query plans. J. Comput. Syst. Sci., 73(3):475-506, 2007. 